Meta

Journal des tradlucteurs

Translators' Journal

\title{
À propos de la lexicographie terminologique...
}

\section{Jacques Lethuillier et Gonia Jarema}

Volume 28, numéro 3, septembre 1983

URI : https://id.erudit.org/iderudit/003483ar

DOI : https://doi.org/10.7202/003483ar

Aller au sommaire du numéro

Éditeur(s)

Les Presses de l'Université de Montréal

ISSN

0026-0452 (imprimé)

1492-1421 (numérique)

Découvrir la revue

Citer cet article

Lethuillier, J. \& Jarema, G. (1983). À propos de la lexicographie

terminologique... Meta, 28(3), 279-283. https://doi.org/10.7202/003483ar d'utilisation que vous pouvez consulter en ligne.

https://apropos.erudit.org/fr/usagers/politique-dutilisation/ 


\section{ÉTUDES \\ TERMINOLOGIQUES \\ ET LINGUISTIQUES}

\section{À PROPOS DE LEXICOGRAPHIE TERMINOLOGIQUE...}

Un colloque sur la lexicographie terminologique s'est tenu les 8,9 et 10 avril dernier à l'Université du Québec à Montréal. Au cours de ces journées, organisées par trois groupes (UQAM, Université Laval et OLF), trois grands thèmes ont été abordés par les conférenciers et les participants : la préparation du travail de lexicographie terminologique, le traitement des notions et la présentation des résultats. L'un des objectifs visés par les organisateurs était de favoriser les échanges de points de vue entre trois catégories de «chercheurs»:

- les spécialistes de domaine qui font de la lexicographie terminologique sans le savoir;

- les terminographes, à la recherche d'une démarche méthodique qui se distingue de celle de la lexicographie générale; et

- les linguistes, pour qui la démarche terminographique ne représente souvent qu'un objet d'étude.

Pour chaque thème se trouvaient réunis un spécialiste de domaine, deux terminographes et un linguiste. Les communications, groupées, étaient suivies de débats animés, le plus souvent fructueux, qui se sont déroulés dans une atmosphère détendue.

Les communications comme les interventions des participants avaient un caractère très pratique. Si les points de vue pouvaient diverger à l'occasion, ils n'étaient pas en général irréconciliables. Dans l'ensemble, des consensus se dégageaient sur la plupart des questions débattues.

Ainsi, il semble clair que, même si les spécialistes du domaine travaillent souvent de façon empirique et ont du mal à reconnaître l'utilité d'une méthode, les terminographes souhaiteraient une plus grande participation de leur part aux travaux terminographiques. Celle-ci pourrait prendre la forme de collaborations, notamment, et elle serait d'autant plus précieuse que la terminologie à explorer véhicule des concepts se situant à un niveau d'abstraction élevé.

Au sujet de la préparation au travail, les terminographes s'entendent sur la nécessité de se familiariser avec le domaine avant d'entreprendre l'exploration méthodique de la terminologie faisant l'objet d'une étude, et en particulier avant de s'attacher à l'élaboration d'un arbre de domaine qui servira de cadre «méthodique». 
Tous sont d'accord aussi sur l'utilité et l'importance de l'arbre, même si chacun s'en fait une idée particulière. L'arbre, en fait, est perçu comme un outil universel, auquel on peut recourir presque à toutes les phases de la démarche terminologique. Les fonctions principales de l'arbre ont été évoquées :

- Aide à la délimitation du champ d'étude; précision de critères d'exclusion.

- Articulation du domaine, visant à faciliter le repérage ainsi que les recoupements dans le cas des travaux de terminologie bilingue.

- Mise en évidence des rapports variés existant entre les notions, utilisables pour traduire la structure du système notionnel par des renvois cohérents, notamment lorsque l'ordre alphabétique est employé pour la présentation.

- Aide à l'élaboration d'un cadre de présentation systématique, lorsque l'ordre alphabétique n'est pas retenu.

- Aide à la composition de définitions formelles, l'arbre permettant de dégager de façon plus rigoureuse les traits distinctifs d'une notion.

- Regroupement des notions par pôles.

- Aide à la présentation des notions sous une forme didactique.

- Outil de recherche dans le cas des dictionnaires automatiques.

- Contrôle de l'exhaustivité du recensement; moyen d'éviter les défauts d'homogénéité dans le repérage.

En bref, pour le terminographe, l'arbre est un instrument d'organisation qui doit demeurer très souple si l'on veut lui faire rendre le plus de services possibles. Il importe de pouvoir lui donner des formes variées, qui soient adaptées à la fonction qu'on lui réserve. À ce sujet, on aurait sans doute intérêt à s'écarter de la structure arborescente stricte et à explorer les possibilités offertes par des modèles déjà couramment utilisés dans d'autres disciplines.

Mais l'engouement des terminographes pour l'arbre n'est pas partagé par les utilisateurs directs des terminologies. Les rapports entre les concepts seraient si clairs pour les locuteurs spécialistes qui les «manient» quotidiennement, que leur visualisation sous la forme de schémas arborescents apparait comme un exercice factice.

Si l'arbre se révèle efficace pour la préparation du travail lexicographique dans de nombreuses circonstances, il ne représente pas cependant une panacée. La terminologie de certaines macro-disciplines ne se laisse pas toujours facilement «encarcaner" dans un arbre de domaine, même si l'on s'éloigne de la forme arborescente stricte. Ces macro-disciplines empruntent une partie plus ou moins importante de leur terminologie à des disciplines autonomes et, à l'occasion, font subir des glissements sémantiques aux termes empruntés. Dans ces situations complexes, qui ne sont pas rares nécessairement, il devient très difficile de définir des critères d'exclusion autrement que de façon empirique, en se fondant sur les besoins de la clientèle-cible.

Au chapitre du traitement des notions, on peut distinguer entre deux grandes orientations : l'option formelle ou l'option didactique. Dans le premier cas, l'outil terminographique s'adresse à des locuteurs spécialistes qui sont familiers avec la terminologie du domaine. Il est utilisé pour la consultation, essentiellement. Dans le second cas, le travail terminographique vise à répondre aux besoins d'un public plus large, englobant notamment les communicateurs, spéciali- 
sés ou non. Le traitement des notions, moins formel, débouche sur une fonction pédagogique. Il doit faciliter l'apprentissage des notions. Cette préoccupation du terminographe se retrouve dans le programme d'information qu'il définit durant la phase de préparation. À ce sujet, des intervenants ont rappelé l'importance du contexte. Au plan sémantique, il permet de préciser la notion en faisant ressortir, par exemple, des traits caractéristiques qui ne sont pas nécessairement distinctifs, ou encore de mettre en relief des caractéristiques extrinsèques. Au plan syntaxique, s'ils sont bien choisis, les contextes apporteront des informations pertinentes sur le comportement syntagmatique des termes, en illustrant les associations qu'ils appellent.

Par ailleurs, on ajoute une dimension à un travail terminographique en accompagnant d'illustrations les textes des articles. Dans le cas de certaines terminologies, lorsque la fonction pédagogique de l'outil terminographique est prépondérante, le recours à l'illustration est même presque indispensable. Il n'est pas toujours aisé de traduire, au moyen d'éléments graphiques, un texte destiné à cerner un concept. Souvent les illustrations ont une valeur particulière. Néanmoins, on doit considérer que l'utilisateur est capable de faire la part des choses et qu'il interprétera d'emblée l'illustration comme la représentation d'un objet particulier de la classe associée au concept défini dans l'article. L'illustration à valeur particulière doit souvent être considérée comme un élément isolé d'une définition en extension se présentant sous forme analogique. En outre, un peu d'imagination permet parfois d'en arriver à illustrer un concept, c'est-à-dire d'en arriver à faire ressortir par le graphisme les caractéristiques essentielles d'une classe d'objets, plutôt que de représenter un objet particulier de cette classe.

Au chapitre de la rédaction des articles, il semble que l'on souhaite que le terminographe devienne plus autonome et prenne l'habitude d'élaborer lui-même des définitions lorsque les circonstances l'exigent. Cela s'explique sans doute par le fait que les contextes définitoires relevés, même s'ils sont tirés d'ouvrages écrits par des spécialistes faisant autorité, n'ont pas toujours une forme idéale et ne mettent pas nécessairement en lumière des traits pertinents de la notion. Dans certaines situations critiques où l'on trouve difficilement des définitions toutes faites et satisfaisantes tant au plan de la forme que du contenu notionnel, le terminographe aurait donc intérêt à rassembler des séries de contextes émanant d'auteurs différents, à se livrer à une analyse rigoureuse des traits évoqués en discours et, à partir des résultats de son analyse, à composer lui-même des définitions en compréhension. Ainsi, l'activité du terminographe évoluerait vers une plus grande autonomie.

En terminologie bilingue, le problème du traitement des synonymes se pose avec une acuité variable selon les disciplines. Présenter l'ensemble des synonymes apparaitra toujours comme une démarche très lourde. Néanmoins, lorsque l'outil terminographique est conçu pour être utilisé à des fins de décodage, aucune liberté n'est laissée au terminographe. Toute la série synonymique doit être enregistrée, selon un ordre déterminé en s'appuyant sur des critères linguistiques et sociolinguistiques précis. On sera plus sélectif dans le cas où la fonction de l'outil terminographique sera restreinte à l'encodage. 
Toujours en terminologie bilingue, des problèmes quasi insurmontables se posent parfois au stade du traitement des notions en raison de l'absence de documentation, voire d'une terminologie, dans l'une des deux langues que l'on se propose d'explorer. Cette absence peut découler du fait qu'un type d'activité industrielle ou technique n'est pas représenté dans les pays appartenant à une communauté linguistique. C'est le cas par exemple pour la terminologie de l'amiante, qui reste embryonnaire en français. Le recours systématique à la néologie s'impose alors. Dans ces circonstances, le terminographe devra posséder une solide formation linguistique.

Au sujet de la présentation générale des résultats, on s'interroge sur le bien-fondé de la recherche d'une certaine uniformité. L'analyse des dictionnaires spécialisés révèle une grande disparité à cet égard. Or, tout semble indiquer qu'elle est voulue, qu'elle reflète la volonté des terminographes de s'adapter aux besoins des utilisateurs et aux caractéristiques de la terminologie du domaine traité, notamment par le choix d'un programme d'information qui tienne compte de la clientèle-cible. Il y a accord de tous les terminographes sur un point, toutefois, c'est le fait que l'on devrait toujours adopter un système cohérent pour marquer, grâce à la typographie, les différentes catégories d'informations apparaissant dans les articles.

Par ailleurs, un aspect particulier de la présentation des résultats retient davantage l'attention, c'est la question de l'ordre dans lequel on fera apparaitre les notions. Deux possibilités s'offrent au terminographe : l'ordre alphabétique ou la présentation systématique.

L'ordre alphabétique, qui présente l'avantage d'accélérer la consultation, n'empêche nullement d'adopter un cadre systématique au moment du repérage et du traitement des notions. Il n'empêche pas non plus de faire ressortir la structure du système notionnel par le truchement de renvois adaptés. On préserve ainsi les avantages de l'ordre systématique sans avoir à en subir le principal inconvénient, c'est-à-dire l'alourdissement de la consultation. En fait la présentation systématique transparaitra derrière l'ordre alphabétique. Ainsi l'ordre alphabétique semble l'emporter. Si la fonction d'apprentissage était prééminente, toutefois, il y aurait intérêt à retenir la présentation systématique.

Dans le cas de certaines terminologies, par ailleurs, la présentation systématique peut s'imposer du fait qu'elle simplifie de façon considérable le traitement des notions. C'est ce qui se passe lorsque le vocabulaire du domaine exploré comporte des termes spécifiques correspondant à des objets dont la fonction est universelle en même temps que l'utilisation très particulière, et dont la dénomination vise à traduire cette particularité. Cerner la notion, en dehors de tout cadre, serait alors très «onéreux». $\mathrm{Si}$, au contraire, le terme apparaît dans un cadre systématique, ce dernier situera la notion de manière précise. La présentation systématique apparaît alors comme une composante du traitement des notions.

Indépendamment du type de présentation choisi, l'insertion des lexies complexes pose au terminographe un problème qui n'est pas toujours simple à résoudre. En terminologie bilingue, le problème se pose avec d'autant plus d'acuité que les composants de sémantisme fort occupent des positions différen- 
tes selon la langue. C'est le cas, par exemple, pour l'anglais et le français. Dans la forme discursive, qui correspond à l'ordre naturel, le noyau syntagmatique et le modificateur occupent des positions inverses.

En fin de colloque, désireux inconsciemment sans doute d'élever le niveau du débat, des linguistes participants aux qualités diverses (gestionnaires, théoriciens et lexicographes) se sont livrés à une simulation réaliste, improvisée et tout à fait réussie d'un type de situation auquel doit faire face assez couramment le terminographe : un désaccord en apparence totalement insurmontable entre spécialistes, au sujet du contenu sémantique d'une notion ou d'un ensemble de notions connexes. En l'occurrence, il s'agissait des notions de notion, de dénomination, de signifié et de signifiant. Le colloque s'est clos sur cette note théorique discordante. Aux linguistes le mot de la fin.

JACQUES LETHUILLIER

GONIA JAREMA 